1 Universidade do Estado do Rio de Janeiro (Uerj), Faculdade de Enfermagem, Programa de Pós-Graduação em Enfermagem - Rio de Janeiro (RJ), Brasil. Orcid: https://orcid. org/0000-0002-56237475

tarcisofeijo@gmail.com

2 Universidade do Estado do Rio de Janeiro (Uerj), Faculdade de Enfermagem, Departamento de Saúde Pública - Rio de Janeiro (RJ), Brasil. Orcid: https://orcid. org/0000-0001-80026830

helenalealdavid@gmail.com

3 Universidade do Estado do Rio de Janeiro (Uerj), Faculdade de Enfermagem - Rio de Janeiro (RJ), Brasil. Orcid: https://orcid. org/0000-0001-69031778

celpcaldas@gmail.com

4 Universidade do Estado do Rio de Janeiro (Uerj), Faculdade de Enfermagem, Programa de Pós-Graduação em Enfermagem - Rio de Janeiro (RJ), Brasil. Orcid: https://orcid. org/0000-0002-65966477

elainelutzmartins@yahoo. com.br

5 Universidade do Estado do Rio de Janeiro (Uerj), Faculdade de Enfermagem, Departamento de Saúde Pública - Rio de Janeiro (RJ), Brasil.

Orcid: https://orcid org/0000-0002-7375-

2751

susanareisesilva@yahoo.

com.br

\section{O acolhimento como estratégia de vigilância em saúde para produção do cuidado: uma reflexão epistemológica}

\author{
User embracement as a surveillance strategy in health care \\ production: an epistemological reflection
}

Tarciso Feijó da Silva', Helena Maria Scherlowski Leal David'², Célia Pereira Caldas ${ }^{\mathbf{3}}$, Elaine Lutz Martins ${ }^{4}$, Susana Reis Ferreira ${ }^{5}$

DOI: $10.1590 / 0103-110420185420$

RESUMO Ensaio de reflexão epistemológica que considerou os sentidos sobre o acolhimento. Os objetivos consistiram em analisar os sentidos sobre o acolhimento em saúde e identificar sua relevância como estratégia de vigilância em saúde para a produção do cuidado na atenção primária. Na prática dos serviços de saúde, o acolhimento envolve relação e produção de saúde. Como instrumento de prática de vigilância em saúde, abarca não apenas a resolução de problemas, mas converge para um cuidado sistematizado e integralizado. Nesse sentido, considerar os significados e sentidos que ele contempla contribui para ressignificá-lo como ferramenta de vigilância em saúde, permitindo considerá-lo como uma estratégia de sustentação do paradigma vigente de produção social da saúde.

PALAVRAS-CHAVE Acolhimento. Atenção Primária à Saúde. Assistência integral à saúde. Vigilância em saúde pública.

ABSTRACT Essay of epistemological reflection that considered the senses of user embracement. The objectives were to analyze the meanings of health care, and to identify its relevance as a strategy of health surveillance for the production of care in primary care. In the practice of the health services, user embracement involves relation and production of health. As an instrument of health surveillance practice, it encompasses not only the resolution of problems, but converges to a systematized and integrated care. In this sense, considering the meanings and senses that it contemplates contributes to re-signifying it as a tool of health surveillance and allowingto consider it as a strategy to support the current paradigm of social production of health.

KEYWORDS User embracement. Primary Health Care. Comprehensive health care. Public health surveillance. 


\section{Introdução}

É na singeleza que reside o segredo de produzir saúde. Para fazer um gesto, dizer uma palavra, basta um. O fato pode ser produzido por uma tecnologia. Mas vale como o outro recebe o gesto, como responde ou corresponde à palavra, como o fato mobiliza sua sensibilidade e a sua ação. $O$ saber se atualiza na interação entre as pessoas. Antes ou independente dessa interação ele é apenas virtualidade. Por esse motivo não é possível fazer saúde para o outro, tratar, curar o outro, mudar seus hábitos, integrá-lo. Saúde é uma co-produção - se trata, se cura e se aprende a cuidar com o outro?.

Rubem Alves, no livro 'Filosofia da ciência', aponta ser relevante que o pesquisador compreenda as questões que envolvem o senso comum para melhor entendimento do que é a ciência. Para o autor, ela é uma metamorfose do senso comum, sendo este necessário para sua existência. Esclarece que é brincando com alguns problemas que se compreende o senso comum e que ser bom em ciência, como ser bom no senso comum, não é saber soluções e respostas já dadas. Estas podem muito bem ser encontradas em livros e receituários. Ser bom em ciência e no senso comum, por sua vez, é ser capaz de inventar soluções. De acordo com o teórico, a ciência, assim como o conhecimento de qualquer tipo, inicia-se quando uma pergunta é disparada, contribuindo para tornar relevante um pensamento que, dada a natureza da sua importância, estabelece uma conversa com a natureza ou com a sociedade ${ }^{2}$.

Nessa perspectiva, ao considerar a gênese do termo acolhimento e as diferentes proposições que convergem para compreender sua relevância para a saúde, assim como as nuances qualitativas associadas a ele nas principais políticas normativas e estudos da área das ciências da saúde, duas questão surgiram: $\mathrm{O}$ acolhimento, no contexto das práticas dos serviços de saúde da atenção primária, alinhado ao Paradigma de Produção Social da Saúde, não teria emergido em uma determinada época para responder às demandas por saúde sob uma perspectiva mais humanística e solidária?; e não poderia ser considerado como uma estratégia de vigilância para a produção do cuidado?

Alerta-se, a priori, que não se tem a intenção de buscar respostas para tais perguntas, antes, porém, pretende-se compartilhar as motivações que contribuíram para a construção deste pensamento. Estas, por sua vez, assentam-se na discussão que envolve os modelos de atenção em saúde, nas diferentes definições sobre o acolhimento e nas dimensões que o envolvem, assim como na relevância dele para a organização dos serviços de saúde, especificamente para os serviços da atenção primária.

Nesse contexto, os objetivos deste ensaio são analisar os sentidos sobre o acolhimento em saúde e identificar sua relevância como estratégia de vigilância em saúde para a produção do cuidado na atenção primária.

\section{A relevância do paradigma}

Os paradigmas, pelo senso comum, podem ser compreendidos como modelos que se tornam um padrão e que são impostos, ou seja, são imposições padronizadas. O 'Dicionário Houaiss da língua portuguesa' define paradigma como uma construção social que dita normas de comportamento que devem ser seguidas, independentemente de essas normas serem benéficas ou prejudiciais, e como um conceito das ciências e da epistemologia vinculado a exemplo, modelo de algo que deve ser seguido ${ }^{3}$.

Para a comunidade científica, paradigma é um modelo ou padrão que orienta a pesquisa. Ele impera sobre as mentes porque institui os conceitos soberanos e a relação lógica que governa, ocultamente, as concepções e as teorias científicas ${ }^{4}$.

Sabe-se que um paradigma são as realizações científicas universalmente reconhecidas que, durante algum tempo, fornecem problemas e soluções modelares para uma comunidade de 
praticantes de uma ciência ${ }^{5}$. São visões de mundo mais abrangentes que teorias, informam das entidades fundamentais da realidade e permitem identificar as entidades que compõem determinada ciência e dizem ao cientistao que procurar e o que esperar².

O paradigma ou matriz disciplinar, como também é chamado pelo teórico Gerard Fourez, é visto, ainda, como a estrutura mental, consciente ou não, que serve para classificar o mundo e poder abordá-lo sob a lente da filosofia da ciência ${ }^{6}$.

Para Kuhn, os paradigmas de uma determinada comunidade científica amadurecida podem ser determinados com relativa facilidade, e abandoná-los é deixar de praticar a ciência que este define ${ }^{5}$. Nesse sentido, um paradigma tem a capacidade de estabelecer uma ruptura com os projetos da vida cotidiana, permitindo eliminar uma série de questões que não são consideradas como pertinentes ${ }^{6}$.

A história da ciência, por exemplo, permite inferir como os atos de observar, medir e interpretar experiências foram radicalmente transformados em razão de diferentes mudanças paradigmáticas ocorridas 5 : a revolução copernicana empreendida por Galileu; a descoberta de Urano realizada por Herschel; a disputa entre Priestley e Lavoisier pela prioridade da descoberta do oxigênio e as mudanças conceituais introduzidas no ato de medir pela mecânica quântica estão entre estas mudanças paradigmáticas ${ }^{5}$.

No campo da saúde, a conotação do termo paradigma, no sentido de movimento ideológico, no entanto, corresponde a um

conjunto de noções, pressupostos e crenças, relativamente compartilhados por um determinado segmento de sujeitos sociais, que serve de referencial para a ação ${ }^{\mathbf{7}(30)}$

\section{As mudanças paradigmáticas na saúde}

No século XIX, a situação da morbimortalidade em todo o mundo era caracterizada pela predominância de doenças infecciosas sobre as demais. Nesse período, tomou corpo a ideia da natureza biológica da doença, deslocando o pensamento causal em saúde do ambiente físico e social para patógenos concretos ${ }^{8}$.

Nesse sentindo, entende-se que a doença teria uma causa única, com um germe originando cada etiologia. Por consequência, saúde era compreendida tão somente como ausência de doença, isto é, ausência de um agravo causado por um germe ${ }^{8}$. O modelo prevalente na maioria dos países capitalistas estruturava-se na visão negativa de saúde vinculando-o à doença e à morte.

Por essa perspectiva, a saúde era percebida apenas como ausência de doença, ganhando dimensão social por meio da visão capitalista emergente. A organização dos serviços começou a ser medicamente definida, passando a ter como objetivo colocar à disposição da população serviços preventivos e curativos reabilitatórios acessíveis?

Esse novo modo de entender a doença exigiu um esforço acadêmico direcionado à mudança do ensino médico, já que não estava preparado para responder às novas evidências e ideias. Entre vários projetos de reformulação do ensino médico que emergiram nos Estados Unidos da América (EUA) e no Canadá, o da Fundação Carneggie obteve êxito e repercussão internacional. Em 1910, a referida Fundação convidou o educador Abraham Flexner, diretor de uma escola secundária de Kentucky, a realizar um estudo sobre a situação das escolas médicas americanas e canadenses. O documento, elaborado após o estudo realizado por Abraham Flexner, ficou conhecido como 'Relatório Flexner' e reforçava o ideário hegemônico da medicina no campo da saúde ${ }^{10}$.

O paradigma flexneriano emergiu a partir desse relatório, sistematizando o ensino e a prática médica, estruturando respostas ao problema das doenças, ou seja, a política sanitária de atenção médica e um conjunto de elementos, tais como: 'individualismo', 'mecanicismo' e 'especialismo'10. Estruturou-se, dessa forma, como resposta para os problemas de saúde: 
a atenção médica e de outros profissionais (dentistas, enfermeiro etc.), medicamentos, exames, equipamentos, entre outros. $\mathrm{O}$ fazer saúde passou a ser entendido como oferta de serviços de saúde, desconsiderando outros aspectos fundamentais"1.

Se, por um lado - para o bem -, o paradigma flexneriano permitiu reorganizar e regulamentar o funcionamento das escolas médicas, por outro - para o mal -, desencadeou um processo terrível de extirpação de todas as propostas de atenção em saúde que não professassem o modelo proposto. O grande mérito - para o bem - do paradigma flexneriano foi a busca da excelência na preparação dos futuros médicos, introduzindo uma salutar racionalidade científica, para o contexto da época. Contudo, ao focar toda a sua atenção nesse aspecto, desconsiderou - para o mal - outros fatores que afetam profundamente os impactos da educação médica na prática profissional e na organização dos serviços de saúde. O paradigma flexneriano, dessa forma, ficou caracterizado por uma concepção mecanicista do processo saúde-doença, pelo reducionismo da causalidade aos fatores biológicos, pelo foco da atenção sobre a doença e sobre o indivíduo, pelo culto à doença, e não à saúde, e pela devoção à tecnologia' ${ }^{12}$.

No Reino Unido, em 1920, Bertrand Dawson elaborava outro relatório que criticava em vários aspectos o Relatório Flexner. Este tinha como propostas uma reorganização dos serviços de saúde, a partir de profissionais generalistas que seriam responsáveis por implementar ações tanto curativas quanto preventivas, com serviços organizados local e regionalmente, por níveis de atenção ${ }^{\mathbf{1 3}}$.

Dawson acreditava que o Estado deveria organizar um sistema de saúde para toda a população. Propôs, pela primeira vez, o esquema conhecido como rede de atenção em saúde, apresentando entre outros os conceitos de: território, populações adscritas, porta de entrada, vínculo/acolhimento, referência e atenção primária como coordenadora do cuidado ${ }^{\mathbf{1 4}}$.

As concepções do documento proposto por
Dawson influenciaram a criação do sistema nacional de saúde britânico que, por sua vez, passou a ser modelo para a reorganização dos sistemas de saúde em vários países do mundo. É importante reiterar que as concepções formuladas por Dawson apontavam para fragilidades no paradigma flexneriano ${ }^{15}$.

No Brasil, a atenção à saúde tem sido historicamente marcada pela predominância da assistência médica curativa e individual e pelo entendimento de saúde como ausência de doença, princípios estes definidos a partir do paradigma flexneriano. A ruptura desse paradigma veio com o ordenamento jurídico-institucional de criação e implantação do Sistema Único de Saúde (SUS), uma vez que o paradigma flexneriano não respondia aos problemas de organização das ações e serviços de saúde de maneira a atender às necessidades de saúde da população ${ }^{16}$.

Com a proposta SUS, além de princípios de organização do sistema (descentralização, regionalização, hierarquização, resolubilidade e complementaridade do setor privado), foram definidos os princípios doutrinários de universalidade de acesso aos serviços de saúde em todos os níveis de assistência; de integralidade da assistência, entendida como um conjunto articulado e contínuo das ações e serviços preventivos e curativos, individuais e coletivos, exigidos para cada caso em todos os níveis de complexidade do sistema; de equidade na assistência à saúde, sem preconceitos ou privilégios de qualquer espécie; e de participação da comunidade ${ }^{17}$.

A construção social de um novo sistema de saúde implicou um processo que exigiu mudanças na concepção do processo saúde-doença. O sentido da mudança foi de uma concepção negativa de saúde para uma concepção positiva de saúde. Esse processo de mudança contribuiu para a concepção de um novo paradigma que ficou conhecido como Paradigma de Produção Social da Saúde. A prática sanitária, nesse contexto de mudança paradigmática da atenção médica, voltou-se para a vigilância em saúde ${ }^{18}$. 
A partir desse novo paradigma, a saúde passou a ser vista como um conceito positivo na medida em que enfatizou os recursos sociais e pessoais, bem como as capacidades físicas de cada indivíduo ${ }^{19}$. O conceito positivo de saúde é caracterizado como a capacidade de ter uma vida satisfatória e proveitosa, confirmada geralmente pela percepção do bem-estar geral ${ }^{20}$. A saúde, a partir dessa nova lógica, não é responsabilidade exclusiva do setor saúde, indo além de um estilo de vida saudável na direção de um bem-estar global ${ }^{19}$.

Segundo o Paradigma de Produção Social da Saúde, salvo a natureza intocada, tudo que existe é produto da ação humana na sociedade, a partir de suas capacidades políticas, ideológicas, cognitivas, econômicas, organizativas e culturais. Uma sociedade poderá, pela sua produção social, acumular saúde ou produzir socialmente enfermidades ${ }^{8}$. O estado de saúde, assim, é um processo que está em permanente transformação e que deve ser entendido como um campo de conhecimento interdisciplinar, assistido na ótica da intersetorialidade, em coerência com o conceito de saúde como expressão da qualidade de vida ${ }^{\mathbf{1 8}}$.

Outros autores ${ }^{21}$ discorrem sobre os princípios que orientam o Paradigma de Produção Social da Saúde. Segundo os autores: o corpo humano é um organismo biológico, psicológico e social, ou seja, recebe informações, organiza, armazena, gera, atribui significados e os transmite, os quais produzem, por sua vez, maneiras de se comportar; saúde e doença são condições que estão em equilíbrio dinâmico, estando codeterminadas por variáveis biológicas, psicológicas e sociais, todas em constante interação; o estudo, diagnóstico, prevenção e tratamento de várias doenças devem considerar as contribuições especiais e diferenciadas dos três conjuntos de variáveis citadas; a etiologia dos estados de doença é sempre multifatorial; a melhor maneira de cuidar de pessoas que estão doentes se dá por ações integradas, realizadas por uma equipe multiprofissional; saúde não é patrimônio ou responsabilidade exclusiva de um grupo de uma especialidade profissional, dessa forma, a investigação e o tratamento não podem permanecer exclusivamente vinculados às especialidades médicas.

O Paradigma de Produção Social da Saúde orientado pela vigilância em saúde assume a responsabilidade de reconstruir as práticas de saúde para dar conta da globalidade do processo saúde-doença. A vigilância em saúde, assim, deve orientar a reformulação das práticas assistenciais e coletivas ${ }^{\mathbf{1 8}}$, devendo estar implicada com as diferentes dimensões que envolvem o processo saúde-doença, tais como: as necessidades sociais de saúde, a atuação com enfoque em grupos de risco, a gestão da agenda para atendimento de demandas programadas e espontâneas e a articulação das atividades de promoção, prevenção, tratamento e reabilitação ${ }^{\mathbf{2 2}}$.

Na prática da vigilância em saúde, destaca-se a relevância do princípio da territorialidade para identificação das prioridades, assim como da utilização adequada dos diferentes recursos existentes para atendimento das necessidades de saúde da população. $\mathrm{O}$ indivíduo é o objetivo final da vigilância em saúde, mas deve ser considerado no contexto da família, da comunidade, do sistema social, do ambiente. Qualquer ação de saúde que se pretenda realizardeverá incidir sobre esse conjunto. Um indivíduo não existe sozinho, isolado. Todo homem é resultado das relações que estabelece ${ }^{23}$.

\section{Acolhimento: significados, sentidos e possibilidades}

Em linhas gerais, poder-se-ia dizer que o acolhimento está presente em todas as relações e encontros que são feitos na vida, mesmo quando se está pouco implicado e envolvido com as relações e com os encontros $^{\mathbf{2 4}}$. Acolher, nos serviços de saúde, significa admitir antes de tudo uma maneira diferente de viver, ou seja, a atitude dos profissionais pode ser a porta de entrada ou de saída dos serviços de saúde' 
O debate que envolve o termo acolhimento a partir do advento da Política Nacional de Humanização (PNH) ganhou nuances qualitativas nas diferentes políticas prescritivas e em outras referências. Os significados atribuídos à palavra ora são convergentes, ora são distintos. Para a PNH, acolher é reconhecer o que o outro traz como legítima e singular necessidade de saúde. $\mathrm{O}$ acolhimento deve comparecer e sustentar a relação entre equipes/serviços e usuários/populações. Como valor das práticas de saúde, o acolhimento é construído de forma coletiva, a partir da análise dos processos de trabalho, e tem como objetivo a construção de relações de confiança, compromisso e vínculo entre as equipes/serviços, trabalhador/equipes e usuário com sua rede socioafetiva. De acordo com a política, a partir da escuta qualificada oferecida pelos trabalhadores às necessidades do usuário, é possível garantir o acesso oportuno desses usuários às tecnologias adequadas às suas necessidades, ampliando a efetividade das práticas de saúde ${ }^{25}$.

Com a PNH, o acolhimento passou a ser considerado como uma das diretrizes de maior relevância ética/estética/política. Ética no que se refere ao compromisso com o reconhecimento do outro, na atitude de acolhê-lo em suas diferenças, suas dores, suas alegrias, seus modos de viver, sentir e estar na vida; estética porque traz para as relações e os encontros do dia a dia a invenção de estratégias que contribuem para a dignificação da vida e do viver e, assim, para a construção da própria humanidade; e política porque implica o compromisso coletivo de envolver-se neste 'estar com', potencializando protagonismos e vida nos diferentes encontros ${ }^{24}$.

O acolhimento pressupõe uma dupla dimensão, pois se de um lado é visto como uma etapa do processo de trabalho, realizado em serviços concretos - em particular no momento da recepção desses serviços -, que estabelece o modo como o serviço faz o seu primeiro contato com a sua clientela, em um processo mútuo de reconhecimento no qual o usuário se reconhece como cliente do serviço e o serviço o reconhece como um usuário com direitos em relação ao serviço realizado, criando suas barreiras e mecanismos de acesso; por outro lado é também visto como uma tecnologia leve do processo intercessor do trabalho em saúde que ocorre em todos os lugares em que se constituem os encontros entre trabalhador e usuários ${ }^{26}$.

Nessa lógica, Franco e colaboradores veem o acolhimento como uma proposta para inversão da lógica de organização e funcionamento dos serviços de saúde, tendo como base o atendimento de todas as pessoas que buscam os serviços de saúde, garantindo acesso universal, reorganização do processo de trabalho com deslocamento das ações do médico para a equipe multiprofissional e qualificação da relação trabalhador-usuário, a partir de parâmetros humanitários que envolvem solidariedade e cidadania ${ }^{27}$.

O acolhimento apresenta-se como uma estratégia fundamental para a qualificação em uma perspectiva de promoção da 'mudança' no processo de trabalho, na reestruturação dos serviços de saúde voltados para a integralidade, a humanização, a equidade e a resolutividade da atenção ${ }^{\mathbf{2 8}}$ e como modo de operar os processos de trabalho em saúde de forma a dar atenção a todos que procuram os serviços de saúde ${ }^{29}$.

Constitui-se como ação que deve ocorrer em todos os locais e momentos do serviço, não devendo limitar-se ao recebimento da demanda espontânea para identificação de risco ou definição de urgências. Diferencia-se de triagem, uma vez que esta última se refere a uma filtragem de quem pode e de quem não pode ser atendido, baseada no que o serviço tem para oferecer, sem considerar as necessidades dos usuários. Acolher, para além, é receber bem, com atenção e disponibilidade para escutar, valorizar as particularidades de cada caso, buscar uma forma de compreendê-lo e solidarizar-se com ele ${ }^{30}$.

O acolhimento é visto como uma das formas de concretizar a humanização das práticas de saúde, não devendo restringir-se somente à escuta, mas avançar para além da fala/expressão verbal; é compreendido como um conjunto 
de medidas, posturas e atitudes dos profissionais de saúde que garanta credibilidade e consideração e pressupõe respeito e solidariedade $^{31}$. Ele emerge como forma de inclusão dos usuários, na medida em que pressupõe que não apenas determinados grupos populacionais (portadores de agravos mais prevalentes e/ ou recortados a partir de ciclos de vida) são objeto privilegiado do trabalho das equipes, mas também as pessoas que apresentam necessidades de saúde e que não estão contempladas nesses critérios. Nessa perspectiva, o acolhimento se revela menos no discurso sobre ele do que nas práticas concretas ${ }^{32}$.

O acolhimento aparece centralmente marcado no território das tecnologias leves, encarnadas nas relações que se estabelecem entre trabalhadores e usuários, nos modos de escutas e filtros, nas maneiras de lidar com o não previsto, nos modos de construção de vínculos, nas formas de sensibilidade do trabalhador, em um certo posicionamento ético situacional ${ }^{32}$. É um espaço-momento de encontro para o reconhecimento de necessidades, acontece com ou sem sala específica, em vários lugares e tempos ${ }^{33}$. Expressa-se na relação estabelecida entre os profissionais de saúde e os (as) usuários (as), mediante atitudes profissionais humanizadoras, que compreendem iniciativas tais como as de: (I) apresentar-se; (II) chamar os (as) usuários(as) pelo nome; (III) prestar informações sobre condutas e procedimentos que devam ser realizados; (IV) escutar e valorizar o que é dito pelas pessoas; (V) garantir a privacidade e a confidencialidade das informações; e (VI) incentivar a presença do (a) acompanhante, entre outras iniciativas semelhantes. De igual forma, o acolhimento também se mostra: (I) na reorganização do processo de trabalho; (II) na relação dos trabalhadores com os modos de cuidar; (III) na postura profissional; e (IV) no vínculo, o que facilita, assim, o acesso aos serviços de saúde ${ }^{34}$. O acolhimento, dessa forma, é compreendido como um recurso, transversal a todas as práticas de saúde ${ }^{35}$.
Atualmente, $\mathrm{o}$ acolhimento vem rompendo com a lógica de organização das unidades de saúde e tem-se configurado na prática dos serviços como um dispositivo para orientação do acesso, priorização de riscos e gestão das agendas de atendimento. Pode ser considerado potente instrumento para prática de vigilância em saúde, desde que tenha um processo sistematizado com foco não somente voltado para resolução de problemas agudos, mas para práticas de cuidados sistematizadas que considerem fatores de risco, vulnerabilidades e possibilidades terapêuticas.

Nesse sentido, poder-se-ia pressupor o acolhimento como uma estratégia de vigilância para produção do cuidado ao considerar não os significados que ele contempla, mas os sentidos que os profissionais e usuários têm reservado ao mesmo no contexto das unidades de saúde. O movimento pela inclusão dos diferentes atores sociais em uma atuação colaborativa que permita a "construção permanente e solidária de laços de cidadania"36(9) expressa um dos mais importantes sentidos do acolhimento.

Estes podem ainda ser expressos pela tomada de decisão dos profissionais em procurar respostas para os problemas dos usuários a partir de fluxos assistenciais que não contemplam todas as demandas da saúde, desenvolvimento de estratégias que conduzam a processos de busca ativa a partir do primeiro contato, adoção de nova configuração do trabalho na perspectiva da multiprofissionalidade, interdisciplinaridade e envolvimento dos usuários, política institucional de construção de redes com projeções intersetoriais, e, principalmente, pelo contato com os usuários permeado pela iniciativa, interesse, envolvimento e empatia. Enquanto projeto de democratização das estruturas de poder e de humanização da assistência à saúde, centrado na primazia da experiência e da sensibilidade. Neste sentido, é importante que o acolhimento assuma o cuidado como uma competência estabelecida e prescrita ${ }^{37}$. 


\section{Justificativas para repensar o acolhimento como estratégia de vigilância para a produção do cuidado}

O acolhimento tem-se apresentado nas práticas dos serviços de saúde como 'modelo de ajuda', em que inclui o atender, o responder, o personalizar, o orientar, o envolver-se, o explorar, o compreender e o agir ${ }^{38}$. Pautado na disponibilidade, na aceitação, na empatia, no diálogo, na cordialidade e na presteza, o acolhimento tem-se configurado como produtor de tranquilidade e segurança para o usuário. Pressupõe a escuta sensível e tensiona aproximação e vinculação observando o significado multidimensional da experiência vivenciada pelos usuários ${ }^{39}$.

Como ferramenta para o processo de educação em saúde, o acolhimento tem-se revelado uma prática de educação solidária e emancipatória, no sentido do exercício e da construção da cidadania, pela escuta e troca de saberes entre conhecimento técnico e popular ${ }^{40}$. Enquanto uma combinação de ações dialógicas, atitudinais e organizativas das práticas de atenção nos centros de saúde, o acolhimento aponta para o desafio cotidiano da alteridade e contribui tanto para melhoria das práticas do cuidado quanto para a legitimação do sistema público de saúde $\mathbf{4 1 , 4 2}^{\text {. }}$

Esse exercício de alteridade é mediado pelo encontro e vai além do processo de reconhecimento e de suas diferenças, singularidades e saberes, antes propõe e desafia a considerar a lógica do outro, o seu 'ponto de vista' e a legitimar o seu conhecimento e as suas necessidades e demandas de saúde. Além disso, desafia a integrar a voz do outro nos processos de escolha e decisão no cotidiano das práticas de cuidado ${ }^{42-44}$.

Diante disso, percebe-se a necessidade de superar o reducionismo biológico, a partir da escuta, da conversa qualificada e compreensão das necessidades de saúde para além do explícito fisicamente, com o propósito de responder adequadamente de forma integrada e integral, fortalecendo também as ações de prevenção ${ }^{38}$.
Nessa perspectiva, o acolhimento não é uma prática isolada, mas um conjunto de práticas que se traduz em atitudes que as pessoas tomam nas interações que se estabelecem no ambiente dos serviços de saúde. A postura adotada pelos profissionais de saúde no acolhimento representa a postura do próprio serviço de saúde, que como ente se constitui 45.

O acolhimento, no contexto das práticas de vigilância em saúde, é visto como um processo que envolve relação e produção de saúde. Nesse sentido, a relação entre os cidadãos trabalhadores de saúde e os cidadãos que buscam atendimento não deve ser uma interação mecânica entre os usuários e agentes, antes deve se constituir em um relacionamento de troca, participação e coprodução. Para isso, há necessidade de mudança de percepção no que diz respeito ao sentir dos profissionais de saúde, o qual tende a ser desagregador, separador e disjuntor. É necessário compreender bem a extensão e a profundidade da ideia de relação, junção, participação, assim como a ideia que se tem do lugar que o outro ocupa no contexto das práticas que são produzidas. Nessa ótica, o acolhimento como processo requer a simultaneidade de várias atividades, e a primeira delas perpassa pela mudança no modo de sentir dos profissionais de saúde, o qual deve se aproximar da cultura humanística. Esta pressupõe considerar que há momentos na vida em que se precisa medir, pesar e contar. $\mathrm{E}$ há, também, momentos em que é preciso levar em conta os sentimentos, as emoções, a subjetividade e a intuição'.

$\mathrm{O}$ acolhimento associa, na forma exata, o discurso da inclusão social, da defesa do SUS a um arsenal técnico extremamente potente, que vai desde a reorganização dos serviços de saúde, a partir do processo de trabalho, até à constituição de dispositivos autoanalíticos e autogestionários, passando por um processo de mudanças estruturais na forma de gestão das unidades de saúde. O resultado esperado é a inversão do paradigma tecnoassistencial ${ }^{27}$. Ele analiticamente pode evidenciar as dinâmicas e os critérios de acessibilidade a que os 
usuários (portadores das necessidades centrais e finalísticas de um serviço) estão submetidos, nas suas relações com 'o quê' os modelos de atenção constituem como verdadeiros campos denecessidades de saúde, para si ${ }^{27}$.

Assim, o que transparece de forma enfática em todo o trabalho de investigação que envolve o acolhimento é sua contemporaneidade, ou seja, a capacidade de se colocar no tempo presente, de mobilizar energias adormecidas, reacender a esperança e colocar em movimento segmentos importantes dos serviços de saúde, como grupos de sujeito que se propõem à construção do novo, a fazer no agora aquilo que é o objetivo no futuro ${ }^{27}$.

\section{Considerações finais}

No paradigma flexneriano, $\mathrm{o}$ atendimento em saúde tende a ser vinculado ao conceito negativo de saúde, e a produção do cuidado tem como foco a doença e a cura. $\mathrm{O}$ acolhimento, nesse mesmo contexto e paradigma, também pode ser considerado como atividade meramente receptiva, isolada e pontual que não envolve vigilância e ações capazes de promover produção do cuidado de forma longitudinal.

Com as mudanças políticas, o contexto social e a construção de um novo sistema de saúde, o paradigma vigente deixou de responder a todas as demandas por saúde, entrando em crise, o que abriu espaço para uma nova perspectiva de análise do contexto social e do cuidado em saúde, possibilitando o surgimento do Paradigma de Produção Social da Saúde. Este analisa o processo saúde-doença com uma abordagem positiva, pautada nas necessidades sociais de saúde, por meio de promoção, prevenção, tratamento e reabilitação em saúde.

Com o advento da PNH, o acolhimento revelou-se como uma diretriz, e diferentes concepções sobre o termo passaram a ser adotadas nas diferentes normativas do Ministério da Saúde e nas pesquisas científicas. As múltiplas concepções sobre o acolhimento convergiram, no contexto do Paradigma de Produção Social da Saúde, para torná-lo potente no cotidiano das práticas de serviços de saúde.

No Paradigma de Produção Social da Saúde, o acolhimento é atravessado por diferentes significados e sentidos que convergem para que ele seja considerado como uma estratégia de vigilância em saúde para a produção do cuidado, pressupondo que seja orientado pela integralidade, pelo conceito positivo de saúde e práticas assistenciais e coletivas de promoção, prevenção, reabilitação e cura.

Nessa ótica, a partir desta reflexão teórica e epistemológica, considera-se necessária a realização de novas pesquisas com vistas a aprofundar a temática sobre o acolhimento, visando identificar nas práticas dos serviços de saúde que sentidos ele tem para os profissionais, se estes compreendem sua relevância como estratégia de vigilância em saúde para a produção do cuidado e para manutenção do Paradigma da Produção Social da Saúde. 


\section{Referências}

1. Zauhy C, Mariotti H. Acolhimento: o pensar, o fazer, o viver. São Paulo: Secretaria Municipal da Saúde; 2002.

2. Alves R. Filosofia da ciência: introdução ao jogo e suas regras. Brasília, DF: Brasiliense; 1981.

3. Houaiss A, Villar MS. Dicionário Houaiss da língua portuguesa: com a nova ortografia da língua portuguesa. Rio de Janeiro: Objetiva; 2009.

4. Morin E. A cabeça bem-feita: repensar a reforma, reformar o pensamento. 8. ed. Rio de Janeiro: Bertrand Brasil; 2003.

5. Kuhn TS. A teoria das revoluções científicas. 5. ed. São Paulo: Perspectiva; 1962.

6. Fourez G. A construção das ciências: introdução à filosofia e à ética das ciências. São Paulo: Universidade Estadual Paulista; 1995.

7. Paim JS, Almeida-Filho N. A crise da saúde pública e a utopia da saúde coletiva. Salvador: Casa da Qualidade; 2000

8. Santos JLF, Westphal MF. Práticas emergentes de um novo paradigma de saúde: o papel da universidade. Estudos Avançados [internet]. 1999 [acesso em 2017 jan 15]; 13(5):71-88. Disponível em: http:// www.scielo.br/scielo.php?script=sci_arttext\&pid =S0103-40141999000100007.

9. Brasil. Conselho Nacional de Educação. Resolução CNE/ CES 2, de 19 de fevereiro de 2002. Institui Diretrizes Curriculares Nacionais do Curso de Graduação em Farmácia. Diário Oficial da União. 19 fev2002.

10. Santos C, Davi J, Martiniano MS, et al. Novo (?) modelo assistencial de saúde. Rev. Pol. Públ. [internet]. 2015 [acesso em 2017 mar 21]; 12(1):73-81. Disponível em: www.revistapoliticaspublicas.ufma.br/site/ download.php?id_publicacao=158.
11. Mendes EV. Mesa redonda: saúde e qualidade de vida. Espaç. Saúde. 1995; 4(4):19-22.

12. Pagliosa FL, Ros MA. The flexner report: for good and for bad. Rev. Bras. Educ. Méd. [internet]. 2008 [acesso em 2017 maio 20]; 32(4):492-499. Disponível em: http://www.scielo.br/scielo.php?script=sci_artt ext\&pid=S0100-55022008000400012.

13. Coelho IB. As propostas de modelos alternativos em saúde. 2006 [acesso em 2017 jun 12]. Disponível em: https://www.nescon.medicina.ufmg.br/biblioteca/ imagem/2147.pdf.

14. Organización Panamericana de la Salud; Organización Mundial de la Salud. Informe Dawson sobre el futuro de los servicios medicos y afines, 1920. Washington, DC: PAHO/OMS; 1964.

15. Lavras C. Atenção primária à saúde e a organização de redes regionais de atenção à saúde no Brasil. Saúde Soc. [internet]. 2011 [acesso em $2016 \mathrm{dez} 15]$; 20(4):867874. Disponível em: http://www.scielo.br/scielo. php?pid=S0104-12902011000400005\&script=sci abstract\&tlng=pt.

16. Scherer MDA, Marino SRA, Ramos FRS. Ruptures and resolutions in the health care model: reflections on the Family Health Strategy based on Kuhn's categories. Interface [internet]. 2004 [acesso em 2016 dez 15]; 9(16):53-66. Disponível em: http:// www.scielo.br/scielo.php?script=sci_arttext\&pid $=$ S1414-32832005000100005.

17. Brasil. Lei $n^{\circ} 8.080$, de 19 de setembro de 1990. Lei Orgânica da Saúde. Dispõe sobre as condições para a promoção, proteção e recuperação da saúde, a organização e o funcionamento dos serviçoes correspondentes e dá outras providências. Diário Oficial da União. 19 Set 1990.

18. Mendes EV. Uma agenda para a saúde. 3. ed. São Paulo: Hucitec; 1999. 
19. Brasil. Ministério da Saúde. Secretaria de Políticas de Saúde. Projeto Promoção da Saúde: as Cartas da Promoção da Saúde. Brasília, DF: Ministério da Saúde; 2002.

20. Nahas MV. Atividade Física, Saúde e Qualidade de vida: Conceitos e sugestões para um estilo de vida ativo. 5. ed. rev. atual. Londrina: Midiograf; 2010.

21. Belloch A, Olabarria B. El modelo biopsicosocial: un marco de referencia necesario para el psicólogo clínico. Ver. Clin. Salud [internet]. 1993 [acesso em 2017 jul2]; 4(2):181-190. Disponível em: http://www.copmadrid.org/webcopm/publicaciones/clinica/1993/ vol2/arti8.htm.

22. Brasil. Conselho Nacional de Secretários de Saúde. Atenção Primária e Promoção da Saúde. Brasília, DF: CONASS, 2007.

23. Campos CEA. Vigilância da saúde no espaço de práticas do PSF. [acesso em 2017 jun 20]. Disponível em: http://www.apmfc.org.br/images/artigos/vigilancia saude.pdf.

24. Brasil. Ministério da Saúde. Secretaria de Atenção à Saúde. Departamento de Atenção Básica. Saúde sexual e saúde reprodutiva. Brasília, DF: Ministério da Saúde; 2010.

25. Brasil. Ministério da Saúde. HumanizaSUS: Política Nacional de Humanização. Brasília, DF: Ministério da Saúde; 2013.

26. Merhy EE, Onocko R. Agir em saúde: um desafio para o público. São Paulo: Hucitec; 1997.

27. Franco TB, Bueno WS, Merhy EE. O acolhimento e os processos de trabalho em saúde: o caso de Betim, Minas Gerais, Brasil. Cad. Saúde Pública [internet]. 1999 [acesso em 2017 maio 16]; (15):345-353. Disponível em: http://www.scielo.br/scielo.php?pid=S0102$-311 X 1999000200019 \&$ script=sci_abstract\&tlng=pt.

28. Brasil. Ministério da Saúde. Secretaria de Atenção à Saúde. Departamento de Atenção Básica. Obesidade. Brasília, DF: Ministério da Saúde; 2006.
29. Brasil. Ministério da Saúde. Secretaria de Atenção à Saúde. Departamento de Atenção Básica. Envelhecimento e saúde da pessoa idosa. Brasília, DF: Ministério da Saúde; 2006.

30. Brasil. Ministério da Saúde. Secretaria de Atenção à Saúde. Departamento de Atenção Básica. Saúde Bucal. Brasília, DF: Ministério da Saúde; 2006.

31. Brasil. Ministério da Saúde. Secretaria de Atenção à Saúde. Departamento de Atenção Básica. Saúde sexual e saúde reprodutiva. Brasília, DF: Ministério da Saúde; 2010.

32. Brasil. Ministério da Saúde. Secretaria de Atenção à Saúde. Departamento de Atenção Básica. Acolhimento à demanda espontânea. Brasília, DF: Ministério da Saúde; 2011.

33. Brasil. Ministério da Saúde. Secretaria de Atenção à Saúde. Departamento de Atenção Básica. Acolhimento à demanda espontânea: queixas mais comuns na Atenção Básica. Brasília, DF: Ministério da Saúde; 2012.

34. Brasil. Ministério da Saúde. Secretaria de Atenção à Saúde. Departamento de Atenção Básica. Atenção ao pré-natal de baixo risco. 1. ed. rev. Brasília, DF: Ministério da Saúde; 2013.

35. Brasil. Ministério da Saúde. Secretaria de Atenção à Saúde. Departamento de Atenção Básica. Departamento de Ações Programáticas Estratégicas. Saúde mental. Brasília, DF: Ministério da Saúde; 2013.

36. Brasil. Ministério da Saúde. Secretaria-Executiva. Núcleo Técnico da Política Nacional de Humanização. HumanizaSUS: Política Nacional de Humanização. A humanização como eixo norteador das práticas de atenção e gestão em todas as instâncias do SUS. Brasília, DF: Ministério da Saúde; 2004.

37. Speroni AV, Menezes RA. Os sentidos do acolhimento: um estudo sobre o acesso à atenção básica em saúde no Rio de Janeiro. Cad. Saúde Coletiva. [internet]. 2014 [acesso em 2017 maio 27]; 22(4):380-385. Disponível em: http://www.scielo.br/scielo.php?pid=S1414- 
$-462 X 2014000400380 \&$ script=sci_abstract\&tlng=pt.

38. Camelo SHH, Angerami ELS, Silva EM, et al. Acolhimento à clientela: estudo em unidades básicas de saúde no município de Ribeirão Preto. Rev. Latino-Am. Enfermagem [internet]. 2000 [acesso em 2016 nov 26]; 8(4):30-37. Disponível em: http://www.scielo.br/scielo.php?pid=S0104-11692000000400005\&script=sci abstract\&tlng=pt.

39. Pelisoli C, Sacco AM, Barbosa ET, et al. Acolhimento em saúde: uma revisão sistemática em periódicos brasileiros. Estud. Psicol. [internet]. 2014 [acesso em 2016 dez 15]; 31(2):225-235. Disponível em: http://www.scielo.br/scielo.php?pid=S0103-166 X2014000200008\&script=sci_abstract\&tlng=pt.

40. Brasil. Ministério da Saúde. Secretaria-Executiva. Núcleo Técnico da Política Nacional de Humanização. Política Nacional de Humanização: acolhimento com avaliação e classificação de risco: um paradigma ético-estético no fazer em saúde. Brasília, DF: Ministério da Saúde; 2004.

41. Teixeira RR. O acolhimento num serviço de saúde entendido como uma rede de conversações. In: Pinheiro R, Mattos RA, organizadores. Construção da integralidade: cotidiano, saberes e práticas em saúde. Rio de Janeiro: IMS/Uerj/Abrasco; 2005.
42. Scholze AS, Duarte Junior CF, Silva YF. Trabalho em saúde e a implantação do acolhimento na atenção básica à saúde: afeto, empatia ou alteridade? Interface [internet]. 2009 [acesso em 2017 fev 23]; 13(31):303-14. Disponível em: http:// www.scielo.br/scielo.php?script=sci_arttext\&pid $=$ S1414-32832009000400006.

43. Cavalcante Filho JB, Vasconcelos EMS, Ceccim RB, et al. Acolhimento coletivo: um desafio instituinte de novas formas de produzir o cuidado. Interface [internet]. 2009 [acesso em 2017 jun 20]; 31(13):315-28. Disponível em: http://www.scielo.br/scielo.php?script=sci arttext\&pid=S1414-32832009000400007.

44. Santos M. A natureza do espaço: técnica e tempo, razão e emoção. São Paulo: USP; 2002.

45. Gomes MCPA, Pinheiro R. Acolhimento e vínculo: práticas de integralidade na gestão do cuidado em saúde em grandes centros urbanos. Interface [internet]. 2005 [acesso em 2017 maio 18]; 9(17):287-302. Disponível em: http://www.scielo.br/scielo.php?pid=S1414$-32832005000200006 \&$ script=sci_abstract.

Recebido em 25/09/2017

Aprovado em 09/03/2018

Conflito de interesses: inexistente

Suporte financeiro: não houve 